\title{
A young woman with hypergammaglobulinemia, distal renal tubular acidosis and some clinical features of polymyositis
}

\author{
K A S Hemachandra ${ }^{1}$, R A Chandrasena ${ }^{1}$ and W A J N Tissera ${ }^{1}$
}

(Index words: renal tubular acidosis, polymyositis, hypokalaemia)

\section{Introduction}

Renal tubular acidosis (RTA) is a disorder of renal acidification of urine due to defective functioning of nephrons. It can be associated with several disease entities including some autoimmune syndromes. We report here a case in which distal RTA is associated with biopsy confirmed polymyositis (PM). So far there is only one case report regarding this association.

\section{Case report}

A 19-year old female was admitted to North Colombo Teaching Hospital with muscle pain and weakness for 1 week. She had no eye involvement or difficulty in swallowing. There was tenderness over the proximal muscles of thighs and arms on both sides. She had proximal muscle weakness with the power of $3 / 5$ in each limb and in neck. Rest of the physical examination was

${ }^{1}$ Medical Unit, Teaching Hospital, Ragama, Sri Lanka.

Correspondence: KASH, e-mail <sampath_hemachandra@yahoo.co.uk>. Received 17 October 2007 and revised version accepted 9 November 2009. Competing interests: none declared. 
unremarkable. There were no other features of connective tissue disorders.

Serum potassium was found to be persistently low (1.5 mmol/1, $1.8 \mathrm{mmol} / 1,2 \mathrm{mmol} / \mathrm{l})$ and serum sodium was $135 \mathrm{mmol} / \mathrm{l}$ and chloride $116 \mathrm{mmol} / \mathrm{l}$. Arterial blood gas analysis revealed $\mathrm{pH}$ of $7.154, \mathrm{pCO}_{2}$ of $3.17 \mathrm{kPa}, \mathrm{pO}_{2}$ of $14.8 \mathrm{kPa}, \mathrm{HCO}_{3}$ of $8.5 \mathrm{mmol} / \mathrm{l}$ and $\mathrm{O}_{2}$ saturation of $98 \%$, suggesting normal anion gap metabolic acidosis. Urine $\mathrm{pH}$ was 8 with no glycosuria. Urinary $\mathrm{pH}$ of more than 5.5 in the presence of severe acidosis suggested RTA.

Investigations showed normal blood counts, blood urea, fasting blood sugar, serum creatinine and serum bilirubin. Serum creatine phosphokinase (CPK) was initially elevated (8723 iu/l). Aspartate aminotransaminase (>150 iu/l) and alanine aminotransaminase (183 iu/l) were elevated. EMG on right quadriceps was compatible with polymyositis. Muscle biopsy showed focal lymphocyte infiltration with muscle fibre destruction and atrophy. Perivascular lymphocyte infiltration was apparent and there was evidence of pericapsular muscle atrophy (Figure 1). These histological features were consistent with polymyositis.



Figure 1. Muscle biopsy showing focal and perivascular lymphocyte infiltration with muscle fibre destruction and atrophy.

Erythrocyte sedimentation rate (ESR) was around 100 in the 1st hour on several occasions. Albumin /globulin ratio was reversed (34/45 g/l) and on electrophoresis, globulin was of polyclonal origin. Serum autoantibody studies rendered no positive result (anti-nuclear factor, double stranded DNA, rheumatoid factor, anti-smooth muscle antibodies and anti Jo-1). Chest X-ray, X-ray KUB, Mantoux test, Coomb's test, echocardiogram and the ultrasound scan of the abdomen did not reveal any abnormal results.

The patient improved markedly after intravenous potassium chloride (oral potassium chloride later) and oral sodium bicarbonate. Steroids or other immuno- suppressives were not given. However CPK level became normal in 7 days. ESR remained high months after the clinical recovery. Oral bicarbonate and potassium replacement were continued and titrated according to blood gas analysis and serum potassium level.

At the beginning we did not consider treating her with immunosuppressive drugs for polymyositis as her condition improved with treatment for RTA. Our plan was to follow up with regard to high ESR, increased gamma globulin and to repeat the muscle biopsy to see whether the muscle inflammation continued. However, the patient stopped attending for follow up after a few months.

\section{Discussion}

RTA is a disorder of renal acidification of urine. In distal RTA there is a secretory defect or a permeability defect of the distal nephron. This defect results in inability to lower the urine $\mathrm{pH}$ to less than 5.5 despite metabolic acidosis. There is associated hypokalaemia. Distal RTA may be a primary disease or associated with autoimmune disorders such as hypergammaglobulinemia, cryoglobulinemia, Sjögren syndrome, thyroiditis, pulmonary fibrosis, chronic active hepatitis, primary biliary cirrhosis, systemic lupus erythematosus, and vasculitis [2].

$\mathrm{PM}$ is an idiopathic, non-infectious, inflammatory myopathy. Our patient met all criteria to diagnose PM. Bohan and Peter criteria of polymyositis include symmetrical proximal muscular weakness, elevated serum muscle enzyme levels, electromyographic evidence of myopathic abnormalities and characteristic findings at muscle biopsy. Severe hypokalaemia can produce muscle paralysis, similar histological features and rhabdomyolysis, causing elevation of muscle enzymes, but markedly elevated PCK level along with typical muscle biopsy findings confirm PM. However improvement of muscle weakness and CPK levels in the absence of immunosuppressive therapy is not explainable.

Distal RTA is rare as an isolated entity, and more commonly it is associated with systemic autoimmune or connective tissue disease. Association of PM with systemic lupus erythematosus, rheumatoid arthritis, Sjögren syndrome, systemic sclerosis and mixed connective tissue disease is well documented. But association of RTA and PM is not described. Persistent high ESR with polyclonal increase in gamma globulin suggests an underlying inflammatory process that is still ill-defined. Taken separately, the patient satisfied the diagnostic criteria of both polymyositis and distal RTA as the cause of proximal myopathy.

\section{Acknowledgement}

We acknowledge Dr. Janaki Fernando and Dr. C. S. B. Godigamuwa for their assistance. 


\section{References}

1. Santh LG, Panchapakesa RC, Jayakumar M, et al. Polymyositis associated with distal renal tubular acidosis (type 1 RTA) a new association? Journal of Indian
Rheumatology Association 2005; 13: 64-6.

2. Asplin JR, Coe FL. Tubular disorders. In: Braunwald E, Fauci AF, Kasper DL, Jameson JL. Harrison's Principals of Internal Medicine. 16th ed. New York: McGraw-Hill. 2005: 1694-702. 